\section{SYNTHESIS OF 1-EPI-} FORTIMICINS A AND B

Sir :

Fortimicins A, $\mathbf{B}^{1,2,3)}$ and their families ${ }^{4,5)}$ are pseudodisaccharide aminocyclitol antibiotics which were found in the culture broth of Micromonospora species. One of the characteristic features of their structures is a novel 1,4-diaminocyclitol named fortamine which is common to the antibiotics. It was of interest to chemically prepare their stereoisomers and compare their antibacterial activity. In this paper we wish to describe the synthesis and activity of 1-epi-fortimicins $\mathbf{A}$ and $\mathbf{B}$.

Tetra-N-benzyloxycarbonylfortimicin $A^{6)}$ (5) was treated with ruthenium tetroxide $\left(\mathrm{RuO}_{2}\right.$ $\mathrm{NaIO}_{4}$ in $\mathrm{CHCl}_{3}$ - water) to give an oxidized product in a low yield (37\%); Rf 0.48 (tlc, $\mathrm{SiO}_{2} /$ toluene - ethyl methyl ketone (EMK), 1: 1), the $\mathrm{Rf}$ value of 5 was 0.30 in the same system, $[\alpha]_{\mathrm{D}}$ $+42.6^{\circ}(c 1.0, \mathrm{MeOH})$. Reduction of 5 with $\mathrm{NaBH}_{4}$ gave tetra-N-benzyloxycarbonyl-1-epifortimicin A (6) (44\%). Deprotection $\left(\mathrm{H}_{2} / \mathrm{Pd}-\mathrm{C}\right)$ of 6 gave 1-epi-fortimicin A (3) after chromatography on Amberlite CG-50 $\left(\mathrm{NH}_{4}{ }^{+}\right.$form) in $74 \%$ yield; $\mathrm{Rf} 0.26$ (tlc, $\mathrm{SiO}_{2} / 2$-propanol - $\mathrm{CHCl}_{3}$ conc. $\left.\mathrm{NH}_{4} \mathrm{OH}, 4: 1: 1\right),[\alpha]_{\mathrm{D}}+71.5^{\circ}\left(c 1.0, \mathrm{H}_{2} \mathrm{O}\right)$. In the pmr spectrum of 3 in basic media, the coupling constants (Table 1) of $J_{3,4}$ was $12 \mathrm{~Hz}$ and $J_{1,2}, J_{2,3}, J_{4,5}, J_{5,8}$, and $J_{1,8}$ were $3 \mathrm{~Hz}$, and a long range coupling $\left(J_{2,6}\right)$ was clearly found, although it could not be measured exactly, confirming that the O-function at the position 6 is axial as with the parent compound. Hence 3 should be 1-epi-fortimicin A. The structure was also defined well its $\mathrm{cmr}$ spectrum (Table 2).

Compound 3 was also synthesized via the 5-Oprotected derivative (9). 5 was benzoylated with $\mathrm{BzCl} / \mathrm{Py}$. to give 2-O-benzoyl derivative (7) $(64 \%)$; Rf 0.32 (tlc, $\mathrm{SiO}_{2} /$ toluene - EMK, 2: 1), $[\alpha]_{\mathrm{D}}$ $+19.0^{\circ}$ (c 1.0, $\mathrm{CHCl}_{3}$ ), ir: 1720 (ester), 1640 (amide I), and $1505 \mathrm{~cm}^{-1}$ (amide II), $\mathrm{pmr}\left(\mathrm{CDCl}_{3}\right.$ ): $\delta 2.92$ (more intense) and 3.01 (each s, total 3, $\mathrm{NCH}_{3}$ ), 3.31 (s, 3, $\mathrm{OCH}_{3}$ ), 5.07 (s, 8, $\mathrm{CH}_{2} \mathrm{Ph} \times 4$ ), 7.27 (s, 20, $\left.\mathrm{CH}_{2} \mathrm{C}_{6} H_{5} \times 4\right)$, and $7.4 \sim 8.0(\mathrm{~m}, 5$, $\mathrm{COC}_{6} \mathrm{H}_{5}$ ). Its debenzyloxycarbonyl derivative (8) showed $\mathrm{Rf} 0.38$ (tlc, $\mathrm{SiO}_{2} / 2$-propanol - $\mathrm{CHCl}_{3}$ conc. $\left.\mathrm{NH}_{4} \mathrm{OH}, 4: 1: 1\right),[\alpha]_{\mathrm{D}}+38.8^{\circ}\left(c 1.0, \mathrm{H}_{2} \mathrm{O}\right)$, and $1725 \mathrm{~cm}^{-1}$ (ester) in its ir. The pmr of 8 showed $\mathrm{H}-2$ proton as a broad singlet $\left(\mathrm{W}_{1 / 2=8 \mathrm{~Hz}}\right)$ at 6.11 ppm (Table 1) confirming its structure. Treat-

Table 1. 100 MHz Pmr parameters for 1-epi-fortimicin A (3), 1-epi-fortimicin B (4), and 2-Obenzoylfortimicin $\mathrm{A}(\mathbf{8})$ in $\mathrm{D}_{2} \mathrm{O}$.

\begin{tabular}{|c|c|c|c|c|c|c|}
\hline & & \multicolumn{2}{|c|}{3} & \multicolumn{2}{|c|}{4} & \multirow{2}{*}{$\begin{array}{c}8 \\
\text { pD } 2.4\end{array}$} \\
\hline & & pD 11.0 & pD 2.5 & pD 11.0 & pD 1.4 & \\
\hline \multicolumn{2}{|l|}{ H-1 } & $3.12 \mathrm{t}$ & & $3.11 \mathrm{t}$ & $3.96 \mathrm{t}$ & $4.01 \mathrm{br} \mathrm{s}$ \\
\hline \multicolumn{2}{|l|}{ H-2 } & $4.39 \mathrm{bt}$ & $4.25 \mathrm{t}$ & $4.20 \mathrm{t}$ & $4.59 \mathrm{t}$ & $6.11 \mathrm{br} \mathrm{s}$ \\
\hline \multicolumn{2}{|l|}{ H-3 } & $3.85 \mathrm{dd}$ & $3.92 \mathrm{dd}$ & $3.40 \mathrm{dd}$ & $3.81 \mathrm{dd}$ & $4.63 \mathrm{dd}$ \\
\hline \multicolumn{2}{|l|}{$\mathrm{H}-4$} & $4.77 \mathrm{dd}$ & $4.63 \mathrm{dd}$ & $2.81 \mathrm{dd}$ & & $5.13 \mathrm{dd}$ \\
\hline \multicolumn{2}{|l|}{ H-5 } & $4.19 t$ & $4.39 t$ & $4.28 \mathrm{t}$ & $4.79 \mathrm{t}$ & $4.24 \mathrm{br} \mathrm{s}$ \\
\hline \multicolumn{2}{|l|}{ H-6 } & $3.84 \mathrm{t}$ & & $3.93 \mathrm{t}$ & $4.36 \mathrm{t}$ & $4.35 \mathrm{br} \mathrm{s}$ \\
\hline \multicolumn{2}{|l|}{$\mathrm{N}-\mathrm{CH}_{3}$} & $3.02 \mathrm{~s}$ & $3.09 \mathrm{~s}$ & $2.37 \mathrm{~s}$ & $2.84 \mathrm{~s}$ & $3.19 \mathrm{~s}$ \\
\hline \multicolumn{2}{|l|}{$\mathrm{O}-\mathrm{CH}_{3}$} & $3.42 \mathrm{~s}$ & $3.48 \mathrm{~s}$ & $3.41 \mathrm{~s}$ & $3.49 \mathrm{~s}$ & $3.52 \mathrm{~s}$ \\
\hline \multicolumn{2}{|l|}{ Gly-CH ${ }_{2}$} & $3.52 \mathrm{~s}$ & $4.06 \mathrm{~s}$ & & & $4.13 \mathrm{~s}$ \\
\hline \multicolumn{2}{|l|}{$\mathrm{H}-\mathrm{1}^{\prime}$} & $4.87 \mathrm{~d}$ & $5.42 \mathrm{~d}$ & $4.88 \mathrm{~d}$ & $5.45 \mathrm{~d}$ & $5.33 \mathrm{~d}$ \\
\hline \multicolumn{2}{|l|}{$6^{\prime}-\mathrm{CH}_{3}$} & $1.04 \mathrm{~d}$ & $1.32 \mathrm{~d}$ & $1.06 \mathrm{~d}$ & $1.35 \mathrm{~d}$ & $1.07 \mathrm{~d}$ \\
\hline \multirow{7}{*}{$\begin{array}{l}\text { The coupling } \\
\text { constants }(\mathrm{Hz})\end{array}$} & $J_{1,2}$ & 3 & 3.5 & 3 & 3.5 & - \\
\hline & $J_{2,3}$ & 3 & 3.5 & 3 & $\sim 3$ & 4 \\
\hline & $J_{3,4}$ & 12 & 7.5 & 10.5 & 11 & 11.5 \\
\hline & $J_{4,5}$ & 3 & $\sim 2.5$ & 3 & $\sim 3$ & 3 \\
\hline & $J_{6,8}$ & 3 & $\sim 3.5$ & 3 & 3.5 & - \\
\hline & $J_{1,6}$ & 3 & $\sim 3.5$ & 3 & 3.5 & - \\
\hline & $J_{1^{\prime}, 2^{\prime}}$ & 3.5 & 3.5 & 3.5 & 3.5 & 3.5 \\
\hline
\end{tabular}


Table 2. Cmr parameters for 1-epi-fortimicin $\mathbf{A}(3)$, 1-epi-fortimicin B (4), and 2-O-benzoylfortimicin $A(8)$ in $\mathrm{D}_{2} \mathrm{O}$.

\begin{tabular}{|c|c|c|c|c|c|}
\hline & \multicolumn{2}{|c|}{3} & \multicolumn{2}{|c|}{4} & \multirow{2}{*}{$\begin{array}{c}8^{*} \\
\text { pD 2.4 }\end{array}$} \\
\hline & pD 11.0 & pD 2.5 & pD 11.0 & pD 1.4 & \\
\hline C-1 & 50.2 & 51.3 & 50.7 & 51.8 & 52.3 \\
\hline C-2 & 69.7 & 65.5 & 65.0 & 61.1 & 68.0 \\
\hline $\mathrm{C}-3$ & 75.4 & 74.0 & 79.8 & 72.0 & 72.5 \\
\hline C-4 & 51.4 & 51.8 & 56.2 & 56.4 & 52.4 \\
\hline C-5 & 70.2 & 70.8 & 70.1 & 64.5 & 71.1 \\
\hline C-6 & 80.0 & 74.0 & 79.1 & 76.2 & 77.5 \\
\hline $\mathrm{N}-\mathrm{CH}_{3}$ & 31.8 & 31.8 & 33.5 & 31.7 & 32.0 \\
\hline $\mathrm{O}-\mathrm{CH}_{3}$ & 56.0 & 56.6 & 57.3 & 57.7 & 57.6 \\
\hline Gly-CO & 176.5 & 168.8 & - & - & 169.0 \\
\hline $\mathrm{Gly}-\mathrm{CH}_{2}$ & 43.4 & 41.2 & - & - & 41.4 \\
\hline $\mathrm{C}-1^{\prime}$ & 99.1 & 92.6 & 99.0 & 91.1 & 98.6 \\
\hline $\mathrm{C}-2^{\prime}$ & 48.6 & 49.1 & 49.0 & 49.0 & 49.6 \\
\hline C-3' & 26.4 & 21.1 & 26.9 & 21.6 & 21.5 \\
\hline C- $4^{\prime}$ & 27.2 & 26.4 & 27.2 & 26.0 & 26.3 \\
\hline C-5' & 74.8 & 68.0 & 74.8 & 71.2 & 70.4 \\
\hline$C-6^{\prime}$ & 50.8 & 49.4 & 50.3 & 49.2 & 51.4 \\
\hline $6^{\prime}-\mathrm{CH}_{3}$ & 18.2 & 17.6 & 18.2 & 15.4 & 15.3 \\
\hline
\end{tabular}

* Signals due to the benzoyl group were found at $167.8(\mathrm{COPh}), 128.9,130.0$ (2), 130.5 (2), and $135.7 \mathrm{ppm}$.

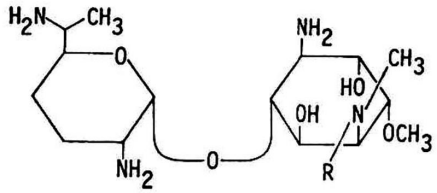

1 Fortimicin $\mathrm{A} \quad \mathrm{R}=\mathrm{COCH}_{2} \mathrm{NH}_{2}$

2 Fortimicin B $R=H$

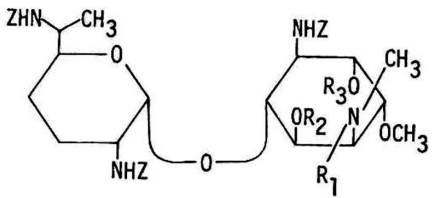

$$
\begin{array}{rlcl} 
& \mathrm{R}_{1} & \mathrm{R}_{2} & \mathrm{R}_{3} \\
5 & \mathrm{COCH}_{2} \mathrm{NHZ} & \mathrm{H} & \mathrm{H} \\
7 & \mathrm{COCH}_{2} \mathrm{NHZ} & \mathrm{H} & \mathrm{Bz} \\
9 & \mathrm{COCH}_{2} \mathrm{NHZ} & \mathrm{THP} & \mathrm{BZ} \\
10 & \mathrm{COCH}_{2} \mathrm{NHZ} & \mathrm{THP} & \mathrm{H} \\
12 & \mathrm{Z} & \mathrm{H} & \mathrm{H}
\end{array}
$$

ment of 7 with 3,4-dihydro- $2 \mathrm{H}$-pyran and $\mathrm{p}$-TsOH in DMF gave $9(70 \%)$; $\mathrm{Rf} 0.56$ (tlc, $\mathrm{SiO}_{2} /$ toluene EMK, 1: 1), which was debenzoylated $\left(\mathrm{NaOCH}_{3}\right.$ in $\left.\mathrm{CH}_{3} \mathrm{OH}\right)$ to give $10(69 \%) ; \mathrm{Rf} 0.30$ and 0.23 (tlc, $\mathrm{SiO}_{2}$ /toluene - EMK, 1: 1). 10 was oxidized

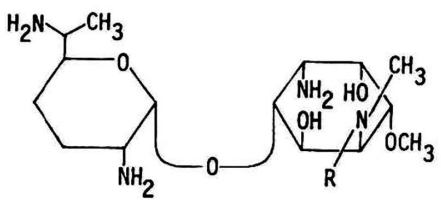

3 T-epi-Fortimicin $A \quad \mathrm{R}=\mathrm{COCH}_{2} \mathrm{NH}_{2}$

4 1-epi-Fortimicin B $\mathrm{R}=\mathrm{H}$

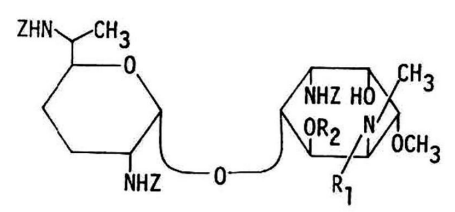

$\mathbf{B z}=$ Benzoyl

THP = Tetrahydropyranyl $\mathbf{Z}=$ Benzyloxycarbonyl in similar way described above, then treated with $\mathrm{NaBH}_{4}$ to give two diastereomers of tetra-Nbenzyloxycarbonyl-5-0-tetrahydropyranyl-1-epifortimicin A, 11a (26\% from 7) and 11b (29\% from 7), 11a: Rf 0.34 (tlc, $\mathrm{SiO}_{2}$ /toluene - EMK, 
Table 3. Antimicrobial spectra of 1-epi-fortimicins A (3) and B (4).

\begin{tabular}{|c|c|c|c|c|}
\hline & \multicolumn{4}{|c|}{$\mathrm{MIC}(\mathrm{mcg} / \mathrm{ml})$} \\
\hline & 3 & FM-A & 4 & FM-B \\
\hline Staphylococcus aureus FDA 209P & 0.78 & 0.2 & 200 & 800 \\
\hline Staphylococcus aureus Smith & 0.39 & 0.2 & 200 & 200 \\
\hline Staphylococcus epidermidis & 0.2 & 0.2 & 800 & 400 \\
\hline Micrococcus luteus ATCC 9341 & 0.39 & 0.2 & 800 & 800 \\
\hline Bacillus subtilis ATCC 6633 & 0.39 & 0.2 & 100 & 100 \\
\hline Escherichia coli NIH JC-2 & 1.56 & 0.78 & 400 & 800 \\
\hline Escherichia coli GN 2411-5 & 3.12 & 1.56 & 800 & 800 \\
\hline Klebsiella pneumoniae 8045 & 0.78 & 0.39 & 400 & 800 \\
\hline Pseudomonas aeruginosa \#1 & 3.12 & 3.12 & 800 & 800 \\
\hline Pseudomonas aeruginosa 145 & & 12.5 & 800 & 800 \\
\hline Serratia marcescens $\mathrm{T}-55$ & 3.12 & 1.56 & 200 & 400 \\
\hline Proteus mirabilis 1287 & & 1.56 & 400 & 800 \\
\hline Proteus vulgaris ATCC 6897 & 1.56 & 1.56 & 400 & 800 \\
\hline Proteus inconstans 39 & & 1.56 & 800 & 800 \\
\hline Escherichia coli KY 8349 & 0.78 & 0.78 & 800 & 800 \\
\hline Pseudomonas aeruginosa KY Z 445 & 12.5 & 6.25 & 800 & 800 \\
\hline Pseudomonas aeruginosa KY 8510 & 6.25 & 6.25 & 800 & 800 \\
\hline Serratia marcescens 1065 & 1.56 & 3.12 & 800 & 800 \\
\hline Providencia 164 & 6.25 & 3.12 & 800 & 800 \\
\hline Escherichia coli KY 8348 & 100 & 100 & 800 & 800 \\
\hline Pseudomonas aeruginosa KY 8563 & 25 & 25 & 800 & 800 \\
\hline Escherichia coli 57R/W677 & 6.25 & 6.25 & 800 & 800 \\
\hline Staphylococcus aureus KY 8970 & & 0.2 & 200 & 400 \\
\hline
\end{tabular}

Assay at pH 7.2, FM: Fortimicin.

2: 1$),[\alpha]_{\mathrm{D}}+63.0^{\circ}$ (c 0.2, $\mathrm{CHCl}_{3}$ ), 11b: Rf 0.28 in the same solvent system, $[\alpha]_{\mathrm{D}}+37.7^{\circ}(c 0.2$, $\mathrm{CHCl}_{3}$ ). 11a and 11b were treated with $70 \%$ acetic acid respectively (room temperature, 1 day and 6 days) to give 6 . Although the stereochemistry of $5,11 \mathrm{a}$ and $11 \mathrm{~b}$ were not identified, they are posturated to be 1-epi-2-ketones (thermodynamically more stable than the corresponding ketones with axial substituents at the position 1). The carbonyl groups, then, presumably are attacked by $\mathrm{NaBH}_{4}$ from the less hindered side of their fortamine rings to form $\alpha$-hydroxyl groups.

The readily available tetra-N-benzyloxycarbonylfortimicin $B^{7)}$ (12) was oxidized and then reduced in the similar way described above to afford tetra-N-benzyloxycarbonyl-1 - epi-fortimicin $\mathrm{B}(13)(18 \%) ; \mathrm{Rf} 0.17$ (tlc, $\mathrm{SiO}_{2} /$ toluene EMK, 3: 1). Deprotection of $13\left(\mathrm{H}_{2} / \mathrm{Pd}-\mathrm{C}\right)$ gave 1-epi-fortimicin B (4) $(99 \%)$; Rf 0.22 (tlc, $\mathrm{SiO}_{2} / 2$-propanol - $\mathrm{CHCl}_{3}$ - conc. $\mathrm{NH}_{4} \mathrm{OH}, 4: 1: 1$ ),
$[\alpha]_{\mathrm{D}}+128.3^{\circ}\left(c 1.0, \mathrm{H}_{2} \mathrm{O}\right)$. The coupling constant of $J_{3,4}$ was $10.7 \mathrm{~Hz}$ and $J_{1,2}, J_{2,3}, J_{4,5}, J_{5,8}$, and $J_{1,8}$ were $2.9 \sim 3.2 \mathrm{~Hz}$ in the basic media, confirming its structure.

Table 3 shows the antibacterial profiles of 1epi-fortimicins A (3) and B (4). Unfortunately 3 does not exhibit improved activity against $E$. coli KY 8348 which is known to produce an inactivating enzyme [AAC(3)-I ${ }^{8}$. Recently, the isolation and characterization of sporaricins ${ }^{\theta, 10)}$, and istamycin $\mathbf{s}^{11)}$ were reported. They are new members of the fortimicin antibiotics containing 2-deoxy- or 2-deoxy-1-epi-fortamine instead of fortamine.

\section{Hiroshi SANo YASUKI MORI Kunikatsu Shirahata*}

Tokyo Research Laboratory, Kyowa Hakko Kogyo Co., Ltd., Machida, Tokyo 194, Japan

* To whom inquiries should be addressed. 
(Received September 1, 1980)

\section{References}

1) Nara, T.; M. Yамamoto, I. KaWAMOTO, K. Takayama, R. OKachi, S. Takasawa, T. Sato \& S. SATO: Fortimicins A and B, new aminoglycoside antibiotics. I. Producing organisms, fermentation and biological properties of fortimicins. J. Antibiotics 30: 533 540, 1977

2) OKachi, R.; S. Takasawa, T. Sato, S. Sato, M. Yamamoto, I. KaWAMOTO \& T. NaRA: Fortimycins $A$ and $B$, new aminoglycoside antibiotics. II. Isolation, physico-chemical and chromatographic properties. J. Antibiotics 30: $541 \sim 551$, 1977

3) Egan, R. S.; R. S. Stanaszek, M. Cirovic, S. L. Mueller, J. TAdanier, J.R. Martin, P.Collum, A.W. Goldstein, R.L. DeVault, A.C. Sinclair, E.E. FAger \& L.A. MItscher: Fortimicins A and $\mathrm{B}$, new aminoglycoside antibiotics. III. Structural identification. J. Antibiotics 30: 552 563, 1977

4) Iida, T.; M. Sato, I. Matsubara, Y. Mori \& K. Shirahata: The structures of fortimicins C, $D$ and KE. J. Antibiotics 32: 1273 1279, 1979

5) Shirahata, K.; G. Shimura, S. Takasawa, T. IIDA \& K. TAKAHASHI: The structure of new fortimicins having double bonds in their purpurosamine moieties. ACS Symposium Series
125: 309 320, 1980

6) Tadanier, J.; J. R. Martin, P. Kurath, A. W. GoldsteIn \& P. JoHNSON：4-N-Acylfortimicins $B$ and the preparation of fortimicin A from fortimicin B. Carbohyd. Res. 79: $91 \sim 102,1980$

7) Sano, H.; T. Sakaguchi \& Y. Mori: Preparation of fortamine and 6-epi-purpurosamine $\mathbf{B}$ from fortimicin B. Bull. Chem. Soc. Jap. 52: $2727 \sim 2728,1979$

8) Sato, S.; T. IIDA, R. OKaChI, K. Shirahata \& T. NARA: Enzymatic acetylation of fortimicin $A$ and seldomycin factor 5 by aminoglycoside 3-acetyltransferase I: [AAC(3)-1] of $E$. coli $\mathrm{KY}$ 8348. J. Antibiotics 30: 1025 1027, 1977

9) Deushi, T.; A. Iwasaki, K. KamiYa, T. KuniedA, T. Mizoguchi, M. Nakayama, H. ITOH, T. MORI \& T. ODA: A new broad-spectrum aminoglycoside antibiotic complex, sporaricin. I. Fermentation, isolation and characterization. J. Antibiotics 32: 173 179, 1979

10) Deushi, T.; M. Nakayama, I. Watanabe, T. Mori, H. Naganawa \& H. Umezawa: A new broad-spectrum aminoglycoside antibiotic complex, sporaricin. III. The structures of sporaricins $A$ and B. J. Antibiotics 32: 187 192, 1979

11) OKaMi, Y.; K. НоTta, M. Yoshida, D. IKeda, S. Kondo \& H. Umezawa: New aminoglycoside antibiotics, istamycins $A$ and B. J. Antibiotics 32: $964 \sim 966,1979$ 